\title{
COMPARATIVE STUDY OF THE LUNG VOLUME INCLUDED IN THE TANGENTIAL FIELD IN BODY CONTOUR BASED AND CT SCAN BASED 2-DIMENSIONAL RADIATION PLANNING IN BREAST CANCER TREATMENT
}

\author{
Sajeev George Pulickal' ${ }^{1}$ Sivarama Krishnan², Girish Babu $M^{3}$ \\ ${ }^{1}$ Associate Professor, Department of Radiotherapy, Government T. D. Medical College, Alappuzha, Kerala, India. \\ 2 Professor, Department of Radiotherapy, Government T. D. Medical College, Alappuzha, Kerala, India. \\ ${ }^{3}$ Assistant Professor, Department of Statistics, Government Arts and Science College, Calicut, Kerala, India.
}

\section{BACKGROUND}

ABSTRACT

Pneumonitis and lung fibrosis are some of the major toxicities of radiation in carcinoma breast, and the volume of lung included in radiation field should be as less as possible. One of the limitations of body contour based 2D (2-dimensional) planning is that we cannot assess the volume of lung included in the radiation field.

Aims and Objectives- To compare the volume of lung included in the tangential radiation field in body contour-based radiation planning with CT scan based 2-dimensional radiation planning in the radiation treatment of carcinoma breast.

\section{MATERIALS AND METHODS}

Post modified radical mastectomy female patients were selected. For radiation planning, we followed 2D planning method by plotting body contour. A single CT scan slice at the level of centre of tangential fields was also taken. Using the treatment planning system, Greatest Perpendicular Distance (GPD) was measured. The percentage lung volume and absolute lung volume were calculated. The observed data were analysed using SPSS software. A p-value of $<0.05$ was considered as significant.

\section{RESULTS}

It was found that the percentage lung volume and the absolute lung volume in CT based planning was less than that in contourbased planning with a difference of median 2.8332 and 79.7783 respectively. Statistical analysis with Wilcoxon signed rank test showed that this difference was significant $(\mathrm{p}<0.0001)$.

\section{CONCLUSION}

The incidence and severity of pneumonitis increase with increasing volume of lung irradiated. GPD can predict the percentage of lung volume irradiated by tangential fields. We can assess GPD using CT scan based 2D planning. This study showed that the percentage lung volume and the absolute lung volume included in the tangential field were significantly less in CT scan based planning method. So, we can reliably use CT scan based 2D planning to reduce the volume of lung irradiated in the treatment of breast cancer in those centres lacking advanced radiation treatment facilities.

\section{KEY WORDS}

Breast Radiation, Radiation Pneumonitis, Greatest Perpendicular Distance.

HOW TO CITE THIS ARTICLE: Pulickal SG, Krishnan S, Babu GM. Comparative study of the lung volume included in the tangential field in body contour based and CT scan based 2-dimensional radiation planning in breast cancer treatment. J. Evolution Med. Dent. Sci. 2018;7(21):2599-2603, DOI: 10.14260/jemds/2018/585

\section{BACKGROUND}

Carcinoma of breast is the most common malignancy of females in developed countries. In India also, it has ranked number one cancer with age adjusted rate as high as 25.8 per 100,000 women. ${ }^{1}$

Radiation therapy has a major role in the breast conservation therapy and in post mastectomy settings. It decreases the risk of local failure by $60 \% .^{2}$ Since pneumonitis and pulmonary fibrosis are some of the major toxicities of chest wall radiation, it is a standard policy to reduce the volume of lung included in radiation field as less as possible. ${ }^{3,4,5,6,7}$

'Financial or Other Competing Interest': None.

Submission 02-04-2018, Peer Review 05-05-2018,

Acceptance 11-05-2018, Published 21-05-2018.

Corresponding Author:

Dr. Sajeev George Pulickal,

Associate Professor, Department of Radiotherapy,

Government T. D. Medical College,

Alappuzha, Kerala, India.

E-mail: pulickan7@gmail.com

DOI: $10.14260 /$ jemds $/ 2018 / 585$

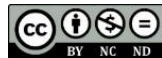

Radiation to chest wall in post mastectomy patients is given using 2 opposing tangential fields. There are various methods to design or plan these radiation fields. This includes 2-Dimensional (2D) planning, simulator planning and 3Dimensional (3D) planning. Simulator is a costly machine, which can be used to plan the tangential fields. 3D planning is done using CT scan images of entire chest. It requires the advanced planning system and linear accelerator.

In a developing country, it is difficult to provide the expensive and advanced treatment facilities to all cancer centres due to financial constraints. Majority of government hospitals in India are having Tele cobalt machine for the radiation treatment. We have Tele cobalt machine in our department and follow 2D planning methods by contouring the shape of chest wall using a wire and plotting it over a paper. In spite of highest care, we cannot reproduce the exact contour of chest wall. Also, we cannot assess the volume of lung included in the radiation field directly from the contour.

The aim of this study was to compare the volume of lung included in the tangential radiation field in body contourbased radiation planning with $\mathrm{CT}$ scan based 2D radiation planning. In CT scan based 2D planning, we take a single slice 
axial CT image of chest and the image is transferred to treatment planning system. The radiation field is planned, and the lung volume included in the field is assessed.

\section{MATERIALS AND METHODS}

This was a descriptive type observational study. Study sample was constituted by post-operative (modified radical mastectomy) female breast cancer patients between the age of 30 and 60 years having WHO performance status $0-1$ with indication for radiotherapy. Patients were selected from our outpatient clinic who were undergoing treatment for breast cancer as per the treatment protocol followed in our department. Patients with contraindications for radiation were excluded.

Sample size was based on the reference study by Bornstein et al, ${ }^{8}$ which included 29 patients. During the study period of 6 months, 30 patients who were willing to participate were included and consent was obtained in proper form. Human Ethical Committee clearance had been obtained for this study.

The study was conducted in two phases. All the 30 patients were included in both phases. First phase was 2D planning by plotting body contour and verification of gantry angles. For contouring, the patients were made to lie supine in the treatment position with ipsilateral arm abducted $>90$ degrees, hand put behind head and head turned to opposite side.

Field borders were drawn over the body and centre of the tangential field marked. Alignment was verified using the positioning of laser fixed in the treatment room.

Body contour at the level of centre of field was taken using thick lead wire and then transferred to a paper. The medial entry point of tangential beam, the midline and the lateral entry point, the mid axillary line were noted in the contour and marked.

Interfield distance (IFD) was measured as the distance between medial and lateral beam entry points and the midpoint of interfield distance was taken as the isocenter. Distance from isocenter to chest wall and gantry angle for medial and lateral tangential beams were determined from the contour. Patient was then taken to the treatment machine and treatment position was reproduced. Gantry angle for the medial and lateral tangential fields were verified.

It is a common finding that with the gantry angle determined by the contour method, the entry point of medial and lateral beams would not be exactly same with the predetermined medial and lateral entry points, i.e. the midline and mid-axillary points. Usually, the lateral entry point will be posterior to mid-axillary line and medial entry point will be slightly lateral to midline. All the beam entry points, i.e. the desired medial midline and lateral mid-axillary points and the newly found entry points were marked with a copper marker.

In the second phase, all the 30 patients were taken for CT based planning. CT scan was done in the Department of Radiodiagnosis.

Treatment positions were reproduced and field center aligned using lasers. A single CT scan slice at the level of center of tangential fields was taken. By this method we got the beam entry points, both the desired midline and midaxillary points and newly found contour-based points in single axial CT scan slice (Fig. 1). CT scan data were transferred to the treatment planning system.

Using treatment planning system, Interfield Distance (IFD) between desired points i.e. midline and mid-axillary points and newly found contour-based points were measured. Tangential beams were put for both desired entry points and newly found contour points and gantry angles measured. Greatest Perpendicular Distance (GPD) was measured from the posterior tangential field edge to parietal pleural surface of anterior chest wall. Percentage and absolute lung volumes were calculated using the equations derived by Bornstein et al in their study-

- $\quad$ Percentage lung volume $=9.5$ (GPD) -7.9

- $\quad$ Absolute lung volume (cc)=122 (GPD) - 94.5

Treatment was given based on the contour-based plan. The dose given for chest wall irradiation was 50 Gy in 25 fractions with 2 Gy per fraction in 5 days/ week. The observed data regarding side affected by the disease, body mass index, chest wall circumference, gantry angles of tangential beam, interfield distance, greatest perpendicular distance etc. were analysed using SPSS software. Results on continuous variables were represented with their median, mean and standard deviation, minimum and maximum. The normality test showed that the GPD values are not following normal. So, we used non-parametric testing method Wilcoxon signed rank test for comparison. A p-value of $<0.05$ was considered as significant.

\section{RESULTS}

\section{Patient Characteristics}

30 female breast cancer patients who underwent modified radical mastectomy and posted for chest wall and regional node radiation treatment were included in this study. Of this, 16 patients were having right-sided breast cancer and 14 patients were having left-sided disease.

Age of the patients included in the study ranged from 30 to 58 yrs.

Considering the body mass index (BMI), the study population included patients having normal weight, overweight and obesity.

Chest wall circumference of the patients in the study population ranged from 73 to $110 \mathrm{~cm}$.

Gantry angle for the tangential chest wall fields obtained from the contour planning method was compared with that obtained from CT planning method.

For right-sided tangential fields, gantry angle for medial tangential field in contour planning method had a mean angle of $52.50^{\circ}$ and ranged from $46^{\circ}$ to $59^{\circ}$.

Gantry angle for medial tangential field in CT based planning method had a mean angle of $62.12^{\circ}$ and ranged from $58^{0}$ to $67^{\circ}$.

Comparing the gantry angles in both planning methods, there was a mean difference of 9.60. Statistical analysis using Wilcoxon signed rank test showed that this difference was significant $(\mathrm{p}<0.0001)$.

For left-sided tangential fields, gantry angle for medial tangential field in contour planning method had a mean angle of $303.78^{\circ}$ and ranged from $298^{\circ}$ to $309^{\circ}$. Gantry angle for medial tangential field in CT planning method had a mean angle of $299.78^{\circ}$ and ranged from $295^{\circ}$ to $305^{\circ}$. 
Comparing the gantry angles in both planning methods, there was a mean difference of $4^{0}$. Statistical analysis using Wilcoxon signed rank test showed that this difference was significant $(\mathrm{p}<0.001)$.

It was found that the tangential fields were steeper in contour-based planning when compared with CT based planning (eg. mean angle in right medial tangential field $52.5^{\circ}$ vs. $62.12^{0}$, contour vs. CT).

Greatest perpendicular distance (GPD)- Greatest perpendicular distance measured from both type of planning methods was compared (Table 1).

The mean GPD in contour-based planning was $1.93 \mathrm{~cm}$ and ranged from $1.13 \mathrm{~cm}$ to $2.4 \mathrm{~cm}$ and median 1.8903 .

The mean GPD in CT based planning method was $1.29 \mathrm{~cm}$ and ranged from $1 \mathrm{~cm}$ to $1.8 \mathrm{~cm}$ and median 1.3012 .

It was found that the GPD in CT based planning was less than that in contour-based planning with a difference of median 0.5891 .

Statistical analysis with Wilcoxon signed rank test showed that this difference was significant $(p<0.0001)$.

\section{Percentage Lung Volume}

Percentage lung volume included in the tangential field was calculated in both methods and compared. The median percentage lung volume included in the tangential field obtained by contour planning method was 9.3542. The median percentage lung volume included in the tangential field obtained by CT planning method was 6.5210 (Table 2). It was found that the percentage lung volume included in the tangential field in CT based planning was less than that in contour-based planning with a difference of median 2.8332 . Statistical analysis with Wilcoxon signed rank test showed that this difference was significant $(p<0.0001)$.

\section{Absolute Lung Volume}

Absolute lung volume included in the tangential field was calculated and compared in both methods. The median absolute lung volume included in the tangential field obtained by contour planning method was 152.7254 . The median absolute lung volume included in the tangential field, obtained by CT planning method was 72.9471 (Table 3). It was found that the absolute lung volume included in the tangential field in CT based planning was less than that in contour-based planning with a difference of median 79.7783 . Statistical analysis with Wilcoxon signed rank test showed that this difference was significant $(p<0.0001)$.

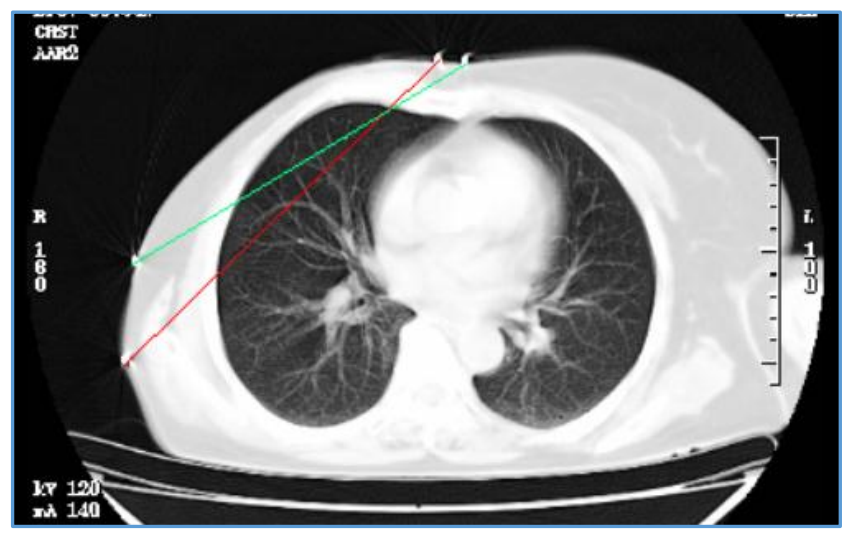

Figure 1. CT Scan Slice at the Level of Central Axis of Tangential Field.

Green-Desired Midline and Mid-Axillary Points. Red-Contour based, Newly Found Point

\begin{tabular}{|c|c|c|c|c|c|c|}
\hline & N & Mean \pm SD & Median & $\begin{array}{c}\text { Interquartile } \\
\text { Range (IQR) }\end{array}$ & Minimum & Maximum \\
\hline GPD. Contour & 30 & $1.9397 \pm 0.4704$ & 1.8903 & 0.48 & 1.13 & 2.40 \\
\hline GPD. CT & 30 & $1.2953 \pm 0.3100$ & 1.3012 & 0.62 & 1.00 & 1.80 \\
\hline \multicolumn{7}{|c|}{ Table 1. GPD (cm) in Contour based and CT based Planning Methods (p<0.0001) } \\
\hline
\end{tabular}

\begin{tabular}{|c|c|c|c|c|c|c|}
\hline & N & Mean \pm SD & Median & $\begin{array}{c}\text { Interquartile } \\
\text { Range (IQR) }\end{array}$ & Minimum & Maximum \\
\hline Percentage lung vol. in contour & 30 & $10.5277 \pm 4.4706$ & 9.3542 & 5.32 & 2.83 & 14.90 \\
\hline Percentage lung vol. in CT & 30 & $4.4057 \pm 2.9454$ & 6.5210 & 4.49 & 1.60 & 9.20 \\
\hline
\end{tabular}

Table 2. Percentage Lung Volume in Contour based and CT based Planning Methods $(p<0.0001)$

\begin{tabular}{|c|c|c|c|c|c|c|}
\hline & N & Mean \pm SD & Median & Interquartile Range (IQR) & Minimum & Maximum \\
\hline Absolute lung vol. in contour & 30 & $142.0380 \pm 57.2974$ & 152.7254 & 61.231 & 43.36 & 198.30 \\
\hline Absolute lung vol. in CT & 30 & $63.5307 \pm 37.8257$ & 72.9471 & 42.3587 & 27.50 & 125.10 \\
\hline
\end{tabular}

\section{DISCUSSION}

The major disadvantage of contour-based planning is that we cannot assess the lung volume included in the tangential field, as it cannot reproduce the patient's chest wall anatomy exactly. It is widely accepted that the incidence and severity of radiation pneumonitis increases with increasing volume of lung irradiated..$^{9,10}$ Bornstein et $\mathrm{al}^{8}$ in their study to determine a simple measurement to predict the amount of lung irradiated introduced the parameters Central Lung Distance (CLD) and Greatest Perpendicular Distance (GPD).

Central Lung Distance [CLD]) is measured from simulator films as the perpendicular distance from the posterior tangential field edge to the posterior part of the anterior chest wall at the center of the field. The greatest perpendicular distance (GPD) is the distance from the posterior tangential field edge to the parietal pleural surface of anterior chest wall and is measured on the CT scan slice corresponding to the tangential field center. The best predictor of the percentage of ipsilateral lung volume treated by the tangential fields was the CLD. A CLD of $1.5 \mathrm{~cm}$ predicted that approximately $6 \%$ of the ipsilateral lung would be included in the tangential field, a CLD of $2.5 \mathrm{~cm}$ approximately $16 \%$ and a CLD of $3.5 \mathrm{~cm}$ approximately $26 \%$ of the ipsilateral lung. In this study, Bornstein et al predicted 
the percentage lung volume and the absolute lung volume by the equations-

- $\quad$ Percentage Lung Volume $=9.5(\mathrm{GPD})_{-} 7.9$

- $\quad$ Absolute Lung Volume = 122 (GPD) _ 94.5

It was observed that, GPD and CLD measure similar distances and both CLD and GPD have better agreement with percentage lung volume than with absolute lung volume. Hence, in the absence of a simulator, GPD can be measured from a single CT scan slice at the central axis and can be used as a predictor of percentage lung volume.

In this present study, we measured GPD for calculating percentage lung volume and absolute lung volume to compare the volume of lung irradiated in contour-based planning method with CT based 2D planning method.

On comparison, it is found that GPD was less in CT based planning than that in contour-based planning. This observation is reflected in the estimation of percentage lung volume and absolute lung volume. Median percentage lung volume in contour planning is 9.3542 . Median percentage lung volume in CT based planning is 6.5210. Difference of median 2.8332 is statistically significant $(\mathrm{p}<0.0001)$. Hence it is evident that using CT based planning method, we can reduce the percentage lung volume irradiated in the tangential fields.

In this study, it is found that median absolute lung volume in contour planning is $\mathbf{1 5 2 . 7 2 5 4}$ and median absolute lung volume in CT based planning is 72.9471. Difference of median 79.7783 is statistically significant $(\mathrm{p}<0.0001)$. Hence, it is clear that using CT based 2D planning we can reduce the absolute lung volume included in the tangential field.

The difference in gantry angle and GPD are some of the factors contributing to the significant increase in the lung volume irradiated in contour-based planning method.

In the present study, it is observed that there is significant difference in gantry angle in contour planning method when compared with CT planning method. The tangential fields are steeper in contour-based planning than that with CT based planning. This leads to increase in GPD and lung volume irradiated in contour-based planning method.

Neal et al ${ }^{11}$ in their study to estimate the volume of lung irradiated during tangential breast radiation using CLD mentioned that a small increase in CLD can dramatically increase the volume of lung actually irradiated. Since CLD and GPD measure the same distance, this is true in the case of GPD also.

Another reason for such a significant difference in lung volume in contour-based planning is the variations in reproducing the treatment position at the time of contouring and at the treatment machine. Bornstein et al in their study found that even though CLD and percentage of ipsilateral lung volume irradiated correlated very well, the mean $90 \%$ prediction interval of $+/-7.1 \%$ indicated some degree of uncertainty. A potential source of error is variation in the patient's position at the simulator and at the CT scanner. In order to reduce this type of error, the same technologist positioned the patient at both procedures.

Here in our study, while using CT planning methods, the entry point of medial tangent beam exactly coincided with midline point in all cases. But in a few cases, the lateral tangential beam entry point deviated posteriorly by $3-5$ millimetres. A probable reason for this variation is the variation in treatment position at the time of contouring, CT scan and treatment. Another reason for this variation is that CT images are taken in the diagnostic CT machine, which is not having a flat couch as that of a planning CT machine or that of a treatment machine.

By using immobilisation devices, we can reduce the patient positioning error. Here, we are not having any immobilisation devices for treating breast cancer patients.

\section{CONCLUSION}

Recent technical advances in radiation planning methods and treatment delivery have made the Radiation Oncologist to delineate the target volume and organs at risk with greater precision and to reduce the radiation dose to organs at risk, especially the ipsilateral lung in the treatment of breast cancer. ${ }^{12}$ In a developing country like India, majority of the cancer centres in government sector are having limited modern treatment facilities like simulator, linear accelerator.

The incidence and severity of pneumonitis increases with increasing volume of lung irradiated. GPD can predict the percentage of lung volume irradiated by tangential fields. We can assess GPD using CT scan based 2D planning. From this study, it is found that the percentage lung volume and absolute lung volume included in the tangential field are significantly less in CT scan based planning method. So, we can reliably use CT scan based 2D planning to reduce the volume of lung irradiated in the treatment of breast cancer in those centres lacking advanced radiation treatment facilities.

\section{ACKNOWLEDGEMENTS}

We are grateful to the Head of Department of Radiodiagnosis for their cooperation to do this study. We are also grateful to our Physicist for his support.

\section{REFERENCES}

[1] Malvia S, Bagadi SA, Dubey US, et al. Epidemiology of breast cancer in Indian women. Asia Pacific Journal of Clinical Oncology 2017;13(4):289-95.

[2] van Dongen JA, Bartelink H, Fentiman IS, et al. Randomized clinical trial to assess the value of breastconserving therapy in stage I and II breast cancer, EORTC 10801 trial. J Natl Cancer Inst Monogr 1992;(11):15-8.

[3] Lind PA, Rosfors S, Wennberg B, et al. Pulmonary function following adjuvant chemotherapy and radiotherapy for breast cancer and the issue of threedimensional treatment planning. Radiother Oncol 1998;49(3):245-54.

[4] Lingos TI, Recht A, Vicini F, et al. Radiation pneumonitis in breast cancer patients treated with conservative surgery and radiation therapy. Int J Radiat Oncol Biol Phys 1991;21(2):355-60.

[5] Rothwell RI, Kelly SA, Joslin CA. Radiation pneumonitis in patients treated for breast cancer. Radiother Oncol 1985;4(1):9-14.

[6] Kimsey FC, Mendenhall NP, Ewald LM. Is radiation treatment volume a predictor for acute or late effects of pulmonary function? Cancer 1994;73(10):2549-55.

[7] Krengli M, Sacco M, Loi G, et al. Pulmonary changes after radiotherapy for conservative treatment of breast cancer: a prospective study. Int J Radiat Oncol Biol Phys 2008;70(5):1460-7. 
[8] Bornstein BA, Cheng CW, Rhodes LM, et al. Can simulation measurements be used to predict the irradiated lung volume in the tangential fields in patients treated for breast cancer? Int J Radiat Oncol Biol Phys 1990;18(1):181-7.

[9] Rothwell RI, Kelly SA, Joslin CA. Radiation pneumonitis in patients treated for breast cancer. Radiother Oncol 1985;4(1):9-14.

[10] Rotstein S, Lax I, Svane G. Influence of radiation therapy on the lung tissue in breast cancer patients: CT-assessed density changes and associated symptoms. Int J Radiat Oncol Biol Phys 1990;18(1):173-80.
[11] Neal AJ, Yarnold JR. Estimating the volume of lung irradiated during tangential breast irradiation using the central lung distance. British J Radiology 1995;68(813):1004-8.

[12] Muren LP, Maurstad G, Hafslund R, et al. Cardiac and pulmonary doses and complication probabilities in standard and conformal tangential irradiation in conservative management of breast cancer. Radiother Oncol 2002;62(2):173-83. 